\title{
Variação no Índice de Massa Corporal em Usuárias de Terapia de Reposição Hormonal
}

\author{
Variations in the Body Mass Index in Users of Hormone Replacement Therapy \\ José Alaércio de Toledo Lima-Junior, Aarão Mendes Pinto-Neto, \\ Lúcia Helena Simões Costa-Paiva, Adriana Orcesi Pedro
}

\begin{abstract}
RESUM0
Objetivo: avaliar os efeitos da terapia de reposição hormonal sobre o indice de massa corporal de mulheres na pós-menopausa.

Casuística e Métodos: foram avaliadas retrospectivamente, por um periodo de três anos, 166 usuárias e 136 não-usuárias de reposição hormonal, acompanhadas no Ambulatório de Menopausa do Centro de Atenção Integral à Saúde da Mulher da Universidade Estadual de Campinas, avaliando-se a variação desse parâmetro ao final de cada ano em relação ao inicial. A análise dos dados foi realizada usando-se o teste $\chi^{2}$, o teste $t$ de Student e o teste de Mann-Whitney para amostras independentes.

Resultados: não foram observadas variações significativas no indice de massa corporal, quando se compararam as usuárias e não-usuárias durante os três anos de observação.

Conclusão: a terapia de reposição hormonal não produziu alterações no índice de massa corporal em mulheres adequadamente acompanhadas durante o seu uso.
\end{abstract}

PALAVRAS-CHAVE: Menopausa. Terapia de reposição hormonal. Índice de massa corporal. Obesidade.

\section{Introdução}

A obesidade é o fator de risco para doença cardiovascular em homens e mulheres identificado há mais tempo, existindo correlação positiva entre ela e a doença coronariana ${ }^{1,2}$. Sua importância deve-se ao fato de que, além de outros aspectos, se trata de fator passivel de prevenção. Aparentemente, a obesidade não é um fator de risco independente para doença cardiovascular, porém está diretamente associada a outros fatores de risco para doença coronariana, como hipertensão arterial, dislipidemia, hiperinsulinemia e resistência à insulina. O risco relativo de doença coronariana chega a ser 3,3 vezes maior em obesos graves ${ }^{3}$.

Departamento de Tocoginecologia da Faculdade de Ciências Médicas da Universidade Estadual de Campinas.

Correspondência:

Aarão Mendes Pinto-Neto

A/C Assessoria Técnica e Científica do CAISM/UNICAMP

Rua Alexander Fleming, 101 - Cidade Universitária

"Zeferino Vaz"

13083-970 - Campinas - SP

Fone: (19) 788-9402
No que diz respeito à população geral, vários fatores estão envolvidos na etiologia da obesidade: fatores genéticos, sexo, fatores psicológicos, socioeconômicos, étnicos e culturais, endócrinos, lesões hipotálamo-hipofisárias e uso de determinados fármacos ${ }^{4}$. A causa mais comum da obesidade é a inatividade, e o aumento proporcional na gordura corporal relacionado com a idade é mais acelerado em individuos sedentários ${ }^{5}$. Em muitos casos, entretanto, não é possivel determinar-se o fator etiológico ${ }^{4}$.

Mulheres de meia-idade tendem a ganhar $0,8 \mathrm{~kg} /$ ano na perimenopausa ${ }^{6}$, efeito este aparentemente não relacionado à deficiência estrogênica ${ }^{7}$. Os possiveis mecanismos que associam o ganho de peso a esse período da vida da mulher são: a mudança do hábito alimentar com tendência à ingestão energética superior ao gasto, a redução do metabolismo basal com propensão a menor gasto energético, além da redução das atividades que exigem motricidade, com tendência ao sedentarismo, todas elas características próprias dessa fase ${ }^{8}$.

Considera-se a terapia de reposição hormonal, 
tanto entre leigos quanto no meio médico, como um dos principais responsáveis pelo ganho de peso na pós-menopausa; porém, não existem evidências científicas que confirmem tal afirmação ${ }^{9}$.

Este fato justificou a realização deste estudo, cujo objetivo foi comparar a variação no índice de massa corporal (IMC) em três anos de observação de mulheres na pós-menopausa, com e sem terapia de reposição hormonal. Desta forma, os resultados poderiam contribuir para uma melhor orientação aos serviços e à população de mulheres com crescente expectativa de vida e que procura hoje, mais e mais, os serviços de saúde, em busca dos beneficios atribuídos à terapia de reposição hormonal.

\section{Pacientes e Métodos}

Este é um estudo de coorte retrospectivo. Para o cálculo do tamanho amostral foi utilizado o estudo de Dallongeville et al. ${ }^{10}$, no qual se avaliou a associação entre a terapia de reposição hormonal e o IMC em um grupo de mulheres, observando-se valores menores de IMC entre as usuárias. Para uma diferença no IMC entre usuárias e nãousuárias de terapia de reposição hormonal de $1,5 \mathrm{~kg} / \mathrm{m}^{2}$, com erro tipo I de 0,05, erro tipo II de 0,20 e desvio-padrão de $4,4 \mathrm{~kg} / \mathrm{m}^{2}$, seriam necessárias 136 mulheres em cada grupo. Foram utilizados dados obtidos dos prontuários médicos de mulheres acompanhadas no Ambulatório de Menopausa do Centro de Atenção Integral à Saúde da Mulher (CAISM) da Universidade Estadual de Campinas (UNICAMP) por um mínimo de três anos. Foram revisados 1.382 prontuários, sendo consideradas adequadas para o estudo 302 mulheres. Destas, 166 usaram terapia de reposição hormonal nos três anos de observação, enquanto 136 não fizeram uso desta terapia.

Os critérios de inclusão foram: mulheres na pós-menopausa com pelo menos um ano de amenorréia e pelo menos dois sintomas clássicos da deficiência estrogênica; pelo menos seis meses de amenorréia, com dosagem de $\mathrm{FSH}$ plasmático maior ou igual a $35 \mathrm{mUI} / \mathrm{ml}^{9}$ e retornos com intervalos de 12 meses ( \pm três meses), durante pelo menos três anos. Os critérios de exclusão foram: o antecedente de uso de terapia de reposição hormonal ou acetato de medroxiprogesterona nos 12 meses anteriores à admissão; presença ou antecedente de neoplasia maligna; contra-indicação absoluta para terapia de reposição hormonal e as pacientes em cujo prontuário não foram encontrados os dados mínimos necessários para o preenchimento da ficha de coleta com precisão.
A variável independente foi o uso de terapia de reposição hormonal. Cerca de 36\% das pacientes utilizaram estrogênios conjugados $(0,625 \mathrm{mg})$ associados a acetato de medroxiprogesterona (5 ou $10 \mathrm{mg}$ ), de maneira continua e por via oral. Aproximadamente 35\% utilizou estrogênios conjugados $(0,625 \mathrm{mg})$ de modo contínuo por via oral, associado a acetato de medroxiprogesterona, 5 ou $10 \mathrm{mg}$, por via oral durante 12 dias/mês. Um quarto das mulheres $(24,2 \%)$ foi tratado com estrogênios conjugados por via oral $(0,625 \mathrm{mg})$, de maneira continua. Destas, $4,2 \%$ utilizaram acetato de medroxiprogesterona $10 \mathrm{mg}$ por via oral, 12 dias/mês, e 0,6\% acetato de medroxiprogesterona, $5 \mathrm{mg}$ ao dia, por via oral, de maneira contínua. Foram consideradas as categorias usuária e não-usuária. A variável dependente considerada foi o índice de massa corporal (IMC) - indicador que relaciona o peso, medido em quilograma, e a altura, medida em metros, ao quadrado ${ }^{11}$. As variáveis de controle foram a idade em anos completos, o tempo de menopausa em meses, o hábito de fumar cigarros, a referência de doenças e drogas utilizadas durante o período de seguimento.

Os grupos "usuárias de terapia de reposição hormonal" e "não-usuárias de terapia de reposição hormonal" foram comparados em cada um dos momentos estudados, ou seja, no início da observação (T0), no ano 1 (T1), no ano 2 (T2) e no ano 3 (T3). Foram calculadas as variações ocorridas em relação à observação inicial, isto é, o dado do ano 1 em relação ao dado inicial (T1 - T0), o dado do ano 2 em relação ao dado inicial (T2 - T0), o dado do ano 3 em relação ao dado inicial (T3 - T0). Utilizou-se o teste $\mathrm{t}$ de Student e teste de MannWhitney para amostras independentes. Utilizouse também o teste $\chi^{2}$ para comparação das características das mulheres entre os grupos.

Foram seguidos os principios estabelecidos pela Resolução n ${ }^{\circ} 196$, de 10 de outubro de 1996 , do Conselho Nacional de Saúde.

\section{Resultados}

As não-usuárias de terapia de reposição hormonal referiram antecedente de doenças mais freqüentemente que as usuárias (50,7\% e 39,2\%, respectivamente; $\mathrm{p}=0,004)$. Não foi encontrada diferença significativa entre as usuárias ou não de terapia de reposição hormonal quanto ao uso ou não de alguma droga, quando considerados os três anos de observação. A grande maioria das mulheres não fumava e não houve diferença na distribuição entre os grupos quanto a esta variável. 
A média de idade das mulheres, distribuída nos grupos de uso ou não de terapia de reposição hormonal, pode ser observada na Tabela 1. Quando se compararam as médias de idade houve diferença estatisticamente significativa (51,4 e 50,0 anos, respectivamente). O tempo de amenorréia não foi diferente entre os grupos $(38,3$ x 29,4 meses).

Tabela 1 - Idade (média de cada grupo) e tempo de amenorréia (T. amen., em meses) no início da observação, segundo uso ou não de terapia de reposição hormonal.

\begin{tabular}{|c|c|c|c|c|c|c|c|}
\hline \multicolumn{8}{|c|}{ TRH } \\
\hline \multirow[t]{2}{*}{ Variável } & \multicolumn{3}{|c|}{ Não } & \multicolumn{3}{|c|}{ Sim } & \multirow[t]{2}{*}{$p$} \\
\hline & Média $\pm \mathrm{DP}$ & Mín & Máx & Média \pm DP & Mín & Máx & \\
\hline Idade & $50,0 \pm 5,4$ & 34 & 65 & $51,4 \pm 4,4$ & 39 & 65 & $0,012^{*}$ \\
\hline T. amen. & $29,4 \pm 34,2$ & 6 & 168 & $38,3 \pm 45,3$ & 6 & 228 & $0,572^{* *}$ \\
\hline
\end{tabular}

Não houve variação significativa na média do IMC das mulheres usuárias de terapia de reposição hormonal nos três anos de acompanhamento, notando-se apenas uma discreta diminuição no primeiro ano de uso e um discreto aumento nos anos subseqüentes. No grupo de não-usuárias observou-se um aumento estatisticamente significativo do IMC quando se verificaram as variações na média entre o segundo e terceiro ano de acompanhamento e a observação inicial de não-usuárias (Tabela 2).

Tabela 2 - Variação média do índice de massa corporal $\left(\mathrm{em} \mathrm{kg} / \mathrm{m}^{2}\right)$ das usuárias e não usuárias de terapia de reposição hormonal de acordo com o tempo de acompanhamento.

\begin{tabular}{|c|c|c|c|c|c|c|c|c|}
\hline \multirow[t]{2}{*}{$\begin{array}{l}\text { Ano de acompanhamento } \\
\text { - usuárias }\end{array}$} & \multicolumn{2}{|c|}{$n$} & \multicolumn{2}{|c|}{$\begin{array}{l}\text { Variação da } \\
\text { Média }\end{array}$} & \multicolumn{2}{|c|}{ DP } & \multicolumn{2}{|c|}{$p$} \\
\hline & TRH & Não & TRH & Não & TRH & Não & TRH & Não \\
\hline 1 & 166 & 135 & $-0,07$ & 0,19 & 1,23 & 1,35 & 0,459 & 0,109 \\
\hline 2 & 165 & 132 & 0,10 & 0,29 & 1,70 & 1,44 & 0,460 & $0,021^{*}$ \\
\hline 3 & 166 & 136 & 0,16 & 0,45 & 1,86 & 1,66 & 0,261 & $0,002^{*}$ \\
\hline
\end{tabular}

DP = Desvio-padrão

*Teste $t$ de Student para amostras pareadas

Com relação ao IMC, notou-se ser discretamente maior no grupo de não-usuárias quando comparado às usuárias de terapia de reposição hormonal. O índice de massa corporal inicial foi de 28,0 $\pm 4,2$ em não-usuárias de terapia e de 27,8 \pm 4,4 nas usuárias, sem diferença estatisticamente significativa. Não houve diferença significativa entre os grupos quando se comparou a variação média do índice de massa corporal das usuárias e não-usuárias de terapia de reposição hormonal nos três anos (Tabela 3).

\section{Discussão}

Nesta avaliação, a terapia de reposição hormonal não mostrou ter grande influência sobre o indice de massa corporal das mulheres em tratamento. As variações observadas não foram diferentes das modificações ocorridas em mulheres não-usuárias da terapia durante os anos de acompanhamento. Existe concordância na literatura a respeito do ganho de peso nas mulheres à época do climatério ${ }^{12}$, o qual parece ser independente da ação hormonal ${ }^{7}$, estando no entanto relacionado a alterações no metabolismo basal.

Tabela 3 - Variação da média do índice de massa corporal (IMC, $\left.\mathrm{kg} / \mathrm{m}^{2}\right)$ de mulheres em uso de terapia reposição hormonal e controles sem TRH antes do início do tratamento(T0) eanualmente por 3 anos(T1-T3).

\begin{tabular}{|c|c|c|c|c|c|c|}
\hline & \multicolumn{6}{|c|}{ Terapia de reposição hormonal } \\
\hline & \multicolumn{3}{|c|}{ Não } & \multicolumn{3}{|c|}{ Sim } \\
\hline & Média & DP & $\mathrm{n}$ & Média & $\mathrm{DP}$ & $\mathrm{n}$ \\
\hline \multicolumn{7}{|c|}{ Média IMC } \\
\hline TO & 28,0 & 4,2 & 136 & 27,8 & 4,4 & 166 \\
\hline $\mathrm{T} 1$ & 28,2 & 4,3 & 135 & 27,8 & 4,3 & 166 \\
\hline $\mathrm{T} 2$ & 28,3 & 4,4 & 132 & 27,9 & 4,5 & 165 \\
\hline $\mathrm{T} 3$ & 28,5 & 4,4 & 136 & 28,0 & 4,5 & 166 \\
\hline \multicolumn{7}{|c|}{ Variação de IMC } \\
\hline $\mathrm{T} 1-\mathrm{TO}^{*}$ & 0,2 & 1,3 & 135 & $-0,07$ & 1,2 & 166 \\
\hline $\mathrm{T} 2-\mathrm{TO}^{*}$ & 0,3 & 1,4 & 132 & 0,10 & 1,7 & 165 \\
\hline T3-T0* & 0,4 & 1,6 & 136 & 0,16 & 1,8 & 166 \\
\hline
\end{tabular}

$\mathrm{DP}=$ Desvio-padrão

* Teste $t$ para amostras independentes - não significativo 
Este estudo avaliou mulheres na pós-menopausa usando formas diferentes de terapia de reposição hormonal, o que, se por um lado condiz com a realidade do ambulatório em questão, por outro lado pode implicar em vieses nos resultados obtidos. Desta forma, os achados podem estar sendo influenciados pelas características das pacientes com diferentes terapias, e não avaliando cada uma separadamente. O tamanho da casuística e a forma como ela foi obtida não nos permitiram fazer o estudo separadamente para cada tipo de terapia de reposição hormonal, já que os subgrupos seriam muito pequenos.

Obtivemos resultados que coincidem com os achados de Reubinoff et al. ${ }^{9}$, que estudaram prospectivamente por um ano 63 mulheres na pósmenopausa recente, usando estrógenos conjugados, 0,625 mg/dia, e acetato de medroxiprogesterona, 2,5 mg/dia. Neste estudo não se observaram diferenças entre usuárias e não-usuárias quanto ao ganho de peso e aumento do IMC no período estudado. Concordamos também com estudo de Jensen et al. ${ }^{13}$ segundo o qual o peso manteve-se inalterado em 136 mulheres na pós-menopausa recebendo terapia de reposição hormonal por um ano, e o de Nachtigal et al. ${ }^{14}$, que encontraram ganho de peso semelhante entre mulheres usando terapia de reposição hormonal ou placebo por dez anos.

Estes resultados, porém, discordam dos de Wing et al. ${ }^{7}$, que, estudando 485 mulheres de meia-idade prospectivamente, encontraram, além de um aumento progressivo no peso e IMC das mulheres, independentemente do estado menopausal, aumento de peso maior em usuárias de terapia hormonal que em não-usuárias. Porém, neste estudo foram avaliadas apenas 32 mulheres usuárias de terapia hormonal, não sendo especificada qual a forma utilizada.

No presente estudo podemos observar que a média de idade das usuárias de terapia de reposição hormonal diferiu das não-usuárias de maneira estatisticamente significativa, porém, julgamos que biologicamente estas diferenças $(51,4$ anos para usuárias e 50,0 para não-usuárias) é pouco importante. Além disso, sabe-se que o tempo de deficiência estrogênica é mais importante que a idade para a incidência de patologias a ela relacionadas ${ }^{6}$.

Este fato, por outro lado, pode refletir uma certa demora no início do uso do tratamento hormonal, ou da procura pelo tratamento, que pode ser justificado pela pouca sintomatologia das mulheres, além do processo educativo longo e lento pelo qual passa esta população, no que diz respeito ao acompanhamento desta fase da vida da mulher.

O dados revelaram que havia mais mulhe- res com algum tipo de patologia no grupo de nãousuárias de reposição hormonal do que no grupo de usuárias, o que está de acordo com os resultados de Matthews et al. ${ }^{15}$, que, estudando prospectivamente 541 mulheres, observaram que as usuárias de terapia de reposição hormonal eram mais saudáveis previamente, ou com menor risco para doença cardiovascular que as não-usuárias desta terapia. Porém, a amostra estudada é representativa da população atendida num ambulatório de referência de acompanhamento do climatério e, em geral, encaminhadas a ele por dificuldades de tratamento em ambulatórios de menor complexidade. Estas pacientes caracterizam-se ainda, pela sua proveniência, na grande maioria das vezes, de classes sociais baixas, com sérias dificuldades de aderência ao tratamento. Inclui também proporção elevada de mulheres com algum tipo de patologia. Em estudo anterior realizado neste mesmo ambulatório, observou-se que mais de um terço das mulheres que iniciaram acompanhamento apresentavam hipertensão ou algum fator de risco para doença cardiovascular ${ }^{16}$.

Apesar de não haver consenso, a terapia de reposição hormonal é considerada hoje como uma terapia associada à diminuição de risco de doença cardiovascular e pode ter como causa de descontinuidade o ganho de peso. Os resultados deste estudo, em que se observou a não-associação entre o uso de terapia de reposição hormonal com o ganho de peso, podem contribuir para a melhora da adesão ao tratamento.

O ganho de peso relacionado a esta fase da vida da mulher, agindo desfavoravelmente nos fatores de risco para doença cardiovascular, merece ações preventivas, que passam pela avaliação e aconselhamento nutricional e combate a hábitos sedentários. E um ambulatório de atendimento à mulher climatérica pode ser a melhor oportunidade, senão a única, para tais ações.

Consideramos que este estudo, apesar de contribuir para um maior conhecimento a respeito do uso da terapia de reposição hormonal, apresenta pontos que necessitam de maior esclarecimento. A associação de cada forma de terapia utilizada com o ganho de peso e os resultados de ações preventivas na tentativa de se evitarem aumentos ao longo do tempo merecem avaliação prospectiva.

Além disso, sabendo-se hoje que a distribuição da gordura corporal está relacionada à doença cardiovascular, de forma que o acúmulo de gordura no abdome está associado a um maior risco da doença, cabe também a avaliação da hipótese de que a proteção contra a doença cardiovascular atribuída à terapia de reposição hormonal está, de alguma forma, associada à distribuição de 
gordura corporal relacionada ao seu uso.

Finalmente concluimos que o ganho de peso é um fator independente do uso da terapia de reposição hormonal; portanto, não justifica a interrupção no tratamento e quando observado, necessita de medidas corretivas de outro tipo ou classe.

\section{SUMMARY}

Purpose: to evaluate the effects of hormone replacement therapy on the body mass index of postmenopausal women. Methods: for this purpose, 166 users and 136 non-users of hormone replacement were evaluated retrospectively during a period of three years. All women were assisted at the Menopause Outpatient Clinic of CAISM - UNICAMP, where the variations in this parameter were evaluated at the end of each year in relation to the initial parameters. The data analysis was performed through $\chi^{2}$ test, Student's t test, and Mann-Whitney test.

Results: we observed no significant variations in the body mass index, when comparing users and non-users during the three years of observation.

Conclusion: hormone replacement therapy did not produce changes in this parameter in women properly assisted during its use.

KEY WORDS: Menopause. Hormone replacement therapy. Body mass index. Obesity.

\section{Referências}

1. Hubert HB, Feinleib M, McNamara PM, Castelli WP. Obesity as an independent risk factor for cardiovascular disease: a 26-year follow-up of participants in the Frammingham heart study. Circulation 1983; 67:968-77.

2. Harlan WR, Landis JR, Flegal KM, Davis CS, Miller ME. Secular trends in body mass in the United States, 1960 - 1980. Am J Epidemiol 1988; 128:1065-74.

3. Manson JE, Colditz GA, Stampfer MJ, et al. A prospective study of obesity and risk of coronary heart disease in women. N Engl J Med 1990; 332:882-9.
4. Grio R, Porpiglia M. Obesity: internal medicine, obstetric and gynecological problems related to overweight. Panminerva Med 1994; 36:138-41.

5. Shangold MM. Role of exercise and nutrition. In: Lobo RA, editor. Treatment of the Postmenopausal Woman: basic and clinical aspects. New York: Raven Press; 1994. p.289-94.

6. Gorodeski GI, Utian WH. Epidemiology and risk factors of cardiovascular disease in postmenopausal women. In: Lobo RA, editor. Treatment of Postmenopausal Woman: basic and clinical aspects. New York: Raven Press; 1994. p.199-221.

7. Wing RR, Mathews KA, Kuller LH, Meilahn EN, Plantinga PL. Weight gain at the time of menopause. Arch Intern Med 1991; 151:99-102.

8. Wang Q, Hassager C, Ravn P, Wang S, Christiansen C. Total and regional body composition changes in early postmenopausal women: age-related or menopause-related? Am Clin Nutr 1994; 60:8438.

9. Reubinoff BE, Wurtman J, Rojansky N, et al. A. Effects of hormone replacement therapy on weight, body composition, fat distribution, and food intake in early postmenopausal women: a prospective study. Fertil Steril 1995; 64:963-8.

10.Dallongeville J, Marecaux N, Isorez D, Zylbergberg G, Fruchart JC, Amouyel P. Multiple coronary heart disease risk factors are associated with menopause and influenced by substitutive hormonal therapy in a cohort of French women. Atherosclerosis 1995; 118:123-33.

11.Kopelman PG. Investigation of obesity. Clin Endocrinol (Oxf) 1994; 41:703-8.

12.Kuskowska-Wolk A, Rossner S. Prevalence of obesity in Sweden: cross-sectional study of a representative adult population. J Intern Med 1990; 227:241-6.

13.Jensen J, Christiansen C, Rodbro P. Oestrogenprogestogen replacement therapy changes body composition in early postmenopausal women. Maturitas 1986; 8:209-16.

14. Nachtigall LE, Nachtigall RH, Nachtigall RD, Beckman EM. Estrogen replacement therapy II: a prospective study in the relationship to carcinoma and cardiovascular and metabolic problems. Obstet Gynecol 1979; 54:74-9.

15.Matthews KA, Kuller LH, Wing RR, Meilahn EN, Plantinga P. Prior to use of estrogen replacement therapy, are users healthier than nonusers? Am J Epidemiol 1996; 143:971-8.

16.Pinto-Neto AM, Paiva LHSC, Petta CA, et al. Fatores de risco para reposição hormonal na menopausa. J Bras Ginecol 1989; 99:387-90. 